\title{
FOURTH-ORDER NONLINEAR EVOLUTION EQUATIONS FOR SURFACE GRAVITY WAVES IN THE PRESENCE OF A THIN THERMOCLINE
}

\author{
SUDEBI BHATTACHARYYA ${ }^{1}$ and K. P. DAS ${ }^{1}$
}

(Received 14 August 1995; revised 21 December 1995)

\begin{abstract}
Two coupled nonlinear evolution equations correct to fourth order in wave steepness are derived for a three-dimensional wave packet in the presence of a thin thermocline. These two coupled equations are reduced to a single equation on the assumption that the space variation of the amplitudes takes place along a line making an arbitrary fixed angle with the direction of propagation of the wave. This single equation is used to study the stability of a uniform wave train. Expressions for maximum growth rate of instability and wave number at marginal stability are obtained. Some of the results are shown graphically. It is found that a thin thermocline has a stabilizing influence and the maximum growth rate of instability decreases with the increase of thermocline depth.
\end{abstract}

\section{Introduction}

There exist a number of papers on nonlinear interaction between surface gravity waves and internal waves. Most of these are concerned with the mechanism of generation of internal waves through nonlinear interaction of surface gravity waves. Coherent three wave interactions of two surface waves and one internal wave have been investigated by Ball [1], Thorpe [22], Watson, West and Cohen [23] and others. Using the theoretical model of Hasselman [12] for incoherent three-wave interaction, Olber and Hertrich [18] have reported a mechanism of generation of internal waves by coupling with surface waves using a three-layer model of the ocean. One of the present authors with Dysthe [9] has investigated a modulational instability mechanism for generation of internal waves using a three-layer model of the ocean.

It is of considerable importance to study the reverse problem, that is, to see how the amplitude of surface gravity waves gets modulated when it interacts with internal

\footnotetext{
${ }^{1}$ Department of Applied Mathematics, University of Calcutta, 92 Acharya Prafulla Chandra Road, Calcutta 700009, INDIA

(C) Australian Mathematical Society, 1997, Serial-fee code 0334-2700/97
} 
waves. Such a study has been made by one of the present authors [3] in which he has investigated the nonlinear evolution of a three-dimensional surface gravity wave packet in a two-layer fluid including the effect of its interaction with internal waves. Similar types of interactions between small-scale surface waves and largescale internal waves in a two-layer fluid has been considered by Rizk and Ko [20]. But they have restricted their analysis to one-dimensional wave packets and to very small density ratios. In deriving the relevant equations they have neglected the conditions of the governing flow at the interface of two fluid layers, and have replaced the internal wave by a surface current produced by it. Resonant interaction between long internal waves and surface gravity waves has been considered by Funakoshi and Oikawa [10] and $\mathrm{Ma}$ [17].

In the present paper we consider the nonlinear evolution of a three dimensional surface gravity wave packet in the presence of a thin thermocline. Considering the importance of the fourth order evolution equation, which was first pointed out by Dysthe [8] and later elaborated by Janssen [14] and considered by many authors ([2, 5$7,11,13,21])$ in studying stability of water waves, two coupled nonlinear evolution equations correct to fourth order in wave steepness are obtained for a surface gravity wave packet in the presence of a thin thermocline. These two coupled equations are reduced to a single equation on the assumption that the space variation of the amplitudes takes place along a line making an arbitrary fixed angle with the direction of propagation of the wave packet. This evolution equation does not remain valid when the resonance condition is satisfied. The resonance occurs when the component of group velocity along the line along which the amplitudes are assumed to vary becomes equal to the phase velocity of the long wavelength internal wave.

The single nonlinear evolution equation is used to study the stability of a uniform surface gravity wave train in the presence of a thin thermocline. Expressions for maximum growth rate of instability and wave number at marginal stability are obtained.

Graphs are plotted showing the variation of the maximum growth rate of the instability against wave steepness for some different values of dimensionless thermocline depth. It is found that a thin thermocline has a stabilising influence on the instability of surface gravity wave packet, and the maximum growth rate of instability decreases with the increase of thermocline depth. The wave number of perturbation $\lambda$ at marginal stability has also been plotted against wave steepness $a_{0}$ showing stable-unstable regions in $\lambda a_{0}$ - space for some different values of thermocline depth and $\theta$, which is the angle between the direction of propagation of the wave and the direction along which the perturbation is given. Graphs are also plotted showing the maximum growth rate of instability against wave steepness for some different values of dimensionless density increase through the thermocline and for some fixed values of dimensionless thermocline depth. 


\section{Basic equations}

We break up the velocity potential $\phi$ and the free surface elevation $\zeta$ into two parts:

$$
\phi=\phi_{0}+\phi_{I}, \quad \zeta=\zeta_{0}+\zeta_{l},
$$

where $\phi_{0}, \zeta_{0}$ are responsible for long wavelength internal waves and $\phi_{l}, \zeta_{1}$ are responsible for surface gravity waves.

For $\phi_{1}$ and $\zeta_{1}$ the equations are the following in which we disregard the existence of the thermocline. They interact with long wavelength internal waves through the nonlinear terms.

$$
\begin{aligned}
& \nabla^{2} \phi_{I}=0, \quad-\infty<Z<\zeta, \\
& \frac{\partial \phi_{I}}{\partial Z}-\frac{\partial \zeta_{I}}{\partial t}=\left(\nabla_{h} \phi\right) \cdot\left(\nabla_{h} \zeta\right), \quad \text { when } Z=\zeta, \\
& \frac{\partial \phi_{I}}{\partial t}+g \zeta_{I}=-\frac{1}{2}(\nabla \phi)^{2}, \quad \text { when } Z=\zeta, \\
& \frac{\partial \phi_{I}}{\partial Z} \rightarrow 0 \quad \text { as } Z \rightarrow-\infty .
\end{aligned}
$$

Here the undisturbed free surface of water has been taken as the $Z=0$ plane; the $Z$-axis points vertically upwards and $\nabla_{h}=\left(\frac{\partial}{\partial x}, \frac{\partial}{\partial y}, 0\right)$.

The governing equations for $\phi_{0}$ and $\zeta_{0}$ are the following, where $\phi_{0}^{\prime}$ is the velocity potential below the thermocline and $w$ is the vertical component of velocity $([9,19])$. The thermocline is assumed to be confined between the two planes $Z=-d-\epsilon$, $Z=-d+\epsilon$, where the thermocline thickness $2 \epsilon$ is small. The terms responsible for long wavelength internal waves are assumed to be sufficiently small so that their product and higher-degree terms can be neglected ([9]).

$$
\begin{aligned}
& \nabla^{2} \phi_{0}=0, \quad-d+\epsilon<Z<\eta, \\
& \nabla^{2} \phi_{0}^{\prime}=0, \quad-\infty<Z<-d-\epsilon, \\
& \left(\frac{\partial \phi_{0}}{\partial Z}\right)_{Z=-d+\epsilon}=\left(\frac{\partial \phi_{0}^{\prime}}{\partial Z}\right)_{Z=-d-\epsilon} \\
& \frac{\partial^{2}}{\partial t^{2}} \nabla^{2} w+N^{2}(Z) \nabla_{h}^{2} w=0, \quad-d-\epsilon<Z<-d+\epsilon, \\
& \frac{\partial \phi_{0}^{\prime}}{\partial Z} \rightarrow 0 \quad \text { as } Z \rightarrow-\infty, \\
& \left(\frac{\partial \phi_{0}}{\partial Z}\right)_{0}-\frac{\partial \zeta_{0}}{\partial t}=\left(\nabla_{h} \phi\right) \cdot\left(\nabla_{h} \zeta\right), \quad \text { when } Z=\zeta, \\
& \frac{\partial \phi_{0}}{\partial t}+g \zeta_{0}=-\frac{1}{2}(\nabla \phi)^{2},
\end{aligned}
$$


where $N^{2}(Z)=-\frac{8}{\rho} \frac{d \rho}{d Z}$, the Brunt-Väisälä frequency, and $\rho$ is the density of water.

Equation (9) is obtained if the Boussinesq approximation is applied, and the long wavelength internal wave is assumed to have sufficiently small amplitude for the nonlinear term to be neglected ([19]).

The thickness of the thermocline being small we shall work with equations obtained by approaching to the limit $\epsilon \rightarrow 0$.

Integrating equation (9) with respect to $Z$ between the limits $-d-\epsilon$ to $-d+\epsilon$ and then proceeding to the limit $\epsilon \rightarrow 0$ we get

$$
\left(\frac{\partial^{4} \phi_{0}}{\partial t^{2} \partial Z^{2}}\right)_{Z=-d}-\left(\frac{\partial^{4} \phi_{0}^{\prime}}{\partial t^{2} \partial Z^{2}}\right)_{Z=-d}=-g \frac{\delta \rho}{\rho} \nabla_{h}^{2}\left(\frac{\partial \phi_{0}}{\partial Z}\right)_{Z=-d},
$$

where $\delta \rho=\rho(-d-0)-\rho(-d+0)$ is the density increase through the thermocline. Proceeding to the limit $\epsilon \rightarrow 0$ in (8) we get

$$
\left(\frac{\partial \phi_{0}}{\partial Z}\right)_{Z=-d}=\left(\frac{\partial \phi_{0}^{\prime}}{\partial Z}\right)_{Z=-d}
$$

So the governing equations for $\phi_{0}, \phi_{0}^{\prime}, \zeta_{0}$ for a thin thermocline are (6), (7), (10)(14).

\section{Derivation of evolution equations}

Following a standard procedure $\phi, \zeta$ given by (1) are expanded as follows ([4]):

$$
\begin{aligned}
& \phi=\phi_{0}+\sum_{n=1}^{\infty}\left(\phi_{n} e^{i n \psi}+\phi_{n}^{*} e^{-i n \psi}\right), \\
& \zeta=\zeta_{0}+\sum_{n=1}^{\infty}\left(\zeta_{n} e^{i n \psi}+\zeta_{n}^{*} e^{-i n \psi}\right),
\end{aligned}
$$

where $\phi_{0}, \phi_{n}, \phi_{n}^{*}$ are functions of $Z, \xi, \eta, \tau$ and $\zeta_{0}, \zeta_{n}, \zeta_{n}^{*}$ are functions of $\xi, \eta, \tau$. Here $\xi=\epsilon\left(x-C_{g} t\right), \eta=\epsilon y, \tau=\epsilon^{2} t, \epsilon$ being a small parameter; $\psi=k x-\omega t, \omega, k$ satisfying the linear dispersion relation

$$
\omega^{2}=g k
$$

for surface - gravity waves; $C_{g}=d \omega / d k$ is the group velocity; $\phi_{0}^{\prime}$ is also a function of $Z, \xi, \eta, \tau$.

Substituting the expansion for $\phi_{I}$ as given by (15) in equation (2) and then equating coefficients of $\exp \operatorname{in} \psi, n=1,2$ on both sides we get the equation

$$
\frac{d^{2} \phi_{n}}{d Z^{2}}-\Delta_{n}^{2} \phi_{n}=0, \quad n=1,2,
$$


where $\Delta_{n}$ is the operator

$$
\Delta_{n}=\left[\left(n k-i \epsilon \frac{\partial}{\partial \xi}\right)^{2}-\epsilon^{2} \frac{\partial^{2}}{\partial \eta^{2}}\right]^{1 / 2} .
$$

The solution of equation (17) satisfying (5) can be put in the form

$$
\phi_{n}=\exp \left(Z \Delta_{n}\right) A_{n},
$$

where $A_{n}$ is a function of $\xi, \eta, \tau$ and the operator $\exp \left(Z \Delta_{n}\right)$ operates on $A_{n}$.

For the sake of convenience we take Fourier transforms of (6), (7) and (10) with respect to $\xi, \eta$ after making a change of independent variables from $x, y, t$ to $\xi, \eta, \tau$.

$$
\begin{aligned}
& \frac{d^{2} \bar{\phi}_{0}}{d Z^{2}}-\epsilon^{2} \bar{k}^{2} \bar{\phi}_{0}=0, \\
& \frac{d^{2} \bar{\phi}_{0}^{\prime}}{d Z^{2}}-\epsilon^{2} \bar{k}^{2} \bar{\phi}_{0}^{\prime}=0, \\
& \frac{d^{2} \bar{\phi}_{0}^{\prime}}{d Z^{2}} \rightarrow 0 \text { as } \quad Z \rightarrow-\infty,
\end{aligned}
$$

where $\bar{\phi}_{0}, \bar{\phi}_{0}^{\prime}$ are Fourier transforms of $\phi_{0}, \phi_{0}^{\prime}$ respectively defined by

$$
\left(\bar{\phi}_{0}, \bar{\phi}_{0}^{\prime}\right)=\frac{1}{(\sqrt{2 \pi})^{2}} \iint_{-\infty}^{\infty}\left(\phi_{0}, \phi_{0}^{\prime}\right) \exp i\left(k_{\xi} \xi+k_{\eta} \eta\right) d \xi d \eta
$$

where $\bar{k}^{2}=k_{\xi}^{2}+k_{\eta}^{2}$. The solution of equation (20) is

$$
\bar{\phi}_{0}=\bar{A}_{0} e^{\epsilon \bar{k} Z}+\bar{B}_{0} e^{-\epsilon \bar{k} Z}
$$

and the solutions of (21) satisfying (22) is

$$
\bar{\phi}_{0}^{\prime}=\bar{C}_{0} e^{\epsilon \bar{k} Z} .
$$

The constants $\bar{A}_{0}, \bar{B}_{0}, \bar{C}_{0}$ appearing in these two equations depend on $k_{\xi}, k_{\eta}, \tau$.

Substituting (15) in the Taylor expanded form of (3) and (4) about $Z=0$ and then equating coefficients of expin $\psi,(n=1,2)$ on both sides, we obtain equations for $A_{1}, \zeta_{1}, A_{2}, \zeta_{2}$. These are the equations (A1), (A2), (A3), (A4) given in the appendix. Next substituting the same expansions (15) for $\phi, \zeta$ on the right-hand side of equations (11), (12) and then keeping only terms independent of $\psi$, we get equations (A5), (A6) given in the appendix. Taking Fourier transforms of (13) and (14) with respect to $\xi, \eta$ and then substituting the solutions for $\bar{\phi}, \bar{\phi}^{\prime}$ given respectively by (24) and (25) we obtain yet two more equations (A7), (A8) given in the appendix. These equations 
(A5) - (A8) determine the quantities $A_{0}, B_{0}, C_{0}, \zeta_{0}$. Eliminating $C_{0}$ between (A7) and (A8) we get the equation (A9) given in the appendix.

Thus we get three sets of equations. The set $\mathrm{I}$ is constituted by equations (Al) and (A2); the set II is constituted by equations (A3) and (A4); and the set III is constituted by equations (A5), (A6) and (A9).

To solve these three sets of equations we make the following perturbation expansion of the quantities $A_{1}, A_{2}, \zeta_{1}, \zeta_{2} \bar{E}, \bar{F}, \zeta_{0}([4])$.

$$
\begin{gathered}
A_{n}=\sum_{j=n}^{\infty} \epsilon^{j} A_{n j}, \quad \zeta_{n}=\sum_{j=n}^{\infty} \epsilon^{j} \zeta_{n j}, \quad(n \geq 1) \\
(\bar{E}, \bar{F})=\sum_{j=n}^{\infty} \epsilon^{j}\left(\bar{E}_{j}, \bar{F}_{j}\right), \quad \zeta_{0}=\sum_{j=n}^{\infty} \epsilon^{j} \zeta_{0 j} .
\end{gathered}
$$

Substituting (26) in the three sets of equations mentioned above and then equating coefficients of various powers of $\epsilon$ on both sides, we get a sequence of equations. From the first (lowest) and second-order equations of (A1) we obtain solutions for $A_{11}$ and $A_{12}$ respectively. Next from the first- and second-order equations of (A3), (A4) we obtain solutions for $A_{22}, \zeta_{22}, A_{23}, \zeta_{23}$. From the first- and second-order equations of (A6) we get solutions for $\bar{\zeta}_{02}, \bar{\zeta}_{03}$; this equation also gives $\zeta_{01}=0$. Finally from the first- and second-order equations of (A5) we get solutions for $F_{2}, F_{3}$; from this equation we also get $F_{1}=0$. All these solutions are given in the appendix.

Substitution of these perturbation solutions in the two equations (A2) and (A7), which have not been used in getting perturbation solutions, produce the following two coupled fourth-order nonlinear evolution equations. Details of the derivation of these two equations are given in the appendix.

$$
\begin{aligned}
i \frac{\partial \zeta}{\partial \tau} & -\frac{1}{8} \frac{\partial^{2} \zeta}{\partial \xi^{2}}+\frac{1}{4} \frac{\partial^{2} \zeta}{\partial \eta^{2}}-\frac{i}{16} \frac{\partial^{3} \zeta}{\partial \xi^{3}}+\frac{3 i}{8} \frac{\partial^{3} \zeta}{\partial \xi \partial \eta^{2}} \\
& =2 \zeta^{2} \zeta^{*}-6 i \zeta \zeta^{*} \frac{\partial \zeta}{\partial \xi}-i \zeta^{2} \frac{\partial \zeta^{*}}{\partial \xi}+\frac{1}{2} \zeta \frac{\partial E}{\partial \xi} \\
-\frac{\partial^{2} E}{\partial \xi^{2}}+4 \gamma k d\left(\frac{\partial^{2}}{\partial \xi^{2}}+\frac{\partial^{2}}{\partial \eta^{2}}\right) E & =-16 \gamma \frac{\partial}{\partial \xi}\left(\zeta \zeta^{*}\right)-4 \frac{\partial}{\partial \xi} H \frac{\partial}{\partial \xi}\left(\zeta \zeta^{*}\right)
\end{aligned}
$$

where $\zeta=\zeta_{11}+\epsilon \zeta_{12}, E=E_{1}+\epsilon E_{2}, H$ is the two-dimensional version of the Hilbert transform given by

$$
\begin{gathered}
H \psi=\frac{1}{2 \pi} \iint_{-\infty}^{\infty} d \xi^{\prime} d \eta^{\prime} \frac{\left(\xi^{\prime}-\xi\right)}{r^{3}} \psi\left(\xi^{\prime}, \eta^{\prime}\right), \\
r=\left[\left(\xi^{\prime}-\xi\right)^{2}+\left(\eta^{\prime}-\eta\right)^{2}\right]^{1 / 2} \quad \text { and } \quad \gamma=\frac{\delta \rho}{\rho} .
\end{gathered}
$$


The two nonlinear evolution equations have been made dimensionless by introducing dimensionless quantities in bars

$$
\bar{\tau}=\omega \tau, \quad \bar{\xi}=k \xi, \quad \bar{\eta}=k \eta, \quad \bar{E}=\frac{2 k^{2}}{\omega} E
$$

and then finally dropping the bars.

Therefore the nonlinear evolution equation of a three-dimensional surface gravity wave packet in presence of a thin thermocline consists of the two coupled equations (27) and (28).

In the absence of a thermocline $\gamma=0$, and therefore (28) gives

$$
\frac{\partial E}{\partial \xi}=4 H \frac{\partial}{\partial \xi}\left(\zeta \zeta^{*}\right)
$$

Substituting this expression for $\partial E / \partial \xi$ in (27) we get the following single nonlinear evolution equation, which is same as the equation (2) of Janssen [14] and (2.20) of Hogan [13] with $\kappa=0$, that is, in the absence of capillarity.

$$
\begin{aligned}
i \frac{\partial \zeta}{\partial \tau} & -\frac{1}{8} \frac{\partial^{2} \zeta}{\partial \xi^{2}}+\frac{1}{4} \frac{\partial^{2} \zeta}{\partial \eta^{2}}-\frac{i}{16} \frac{\partial^{3} \zeta}{\partial \xi^{3}}+\frac{3 i}{8} \frac{\partial^{3} \zeta}{\partial \xi \partial \eta^{2}} \\
& =2 \zeta^{2} \zeta^{*}-6 i \zeta \zeta^{*} \frac{\partial \zeta}{\partial \xi}-i \zeta^{2} \frac{\partial \zeta^{*}}{\partial \xi}+2 \zeta H \frac{\partial}{\partial \xi}\left(\zeta \zeta^{*}\right)
\end{aligned}
$$

We shall now derive a single nonlinear evolution equation from the two coupled equations (27) and (28) on the assumption that space variation of the amplitudes takes place along a direction which makes an angle $\theta$ with the direction of propagation of the wave. Such an equation is suitable for a stability analysis of a uniform wave train with respect to a plane wave perturbation given in a direction making an angle $\theta$ with the direction of propagation of the wave.

Transforming the horizontal co-ordinates $\xi, \eta$ into the new co-ordinates $\xi^{\prime}, \eta^{\prime}$ by the relations

$$
\xi^{\prime}=\xi \cos \theta+\eta \sin \theta, \quad \eta^{\prime}=-\xi \sin \theta+\eta \cos \theta
$$

and then assuming that $\zeta, E$ depend on $\xi^{\prime}$ only and not on $\eta^{\prime}$, the evolution equations (27) and (28) get transformed into the following two coupled equations.

$$
\begin{aligned}
i \frac{\partial \zeta}{\partial \tau} & -\left(\frac{1}{8} \cos ^{2} \theta-\frac{1}{4} \sin ^{2} \theta\right) \frac{\partial^{2} \zeta}{\partial \xi^{\prime 2}}-i\left(\frac{1}{16} \cos ^{3} \theta-\frac{3}{8} \cos \theta \sin ^{2} \theta\right) \frac{\partial^{3} \zeta}{\partial \xi^{\prime 3}} \\
& =2 \zeta^{2} \zeta^{*}-6 i \cos \theta \zeta \zeta^{*} \frac{\partial \zeta}{\partial \xi^{\prime}}-i \cos \theta \zeta^{2} \frac{\partial \zeta^{*}}{\partial \xi^{\prime}}+\frac{1}{2} \cos \theta \zeta \frac{\partial E}{\partial \xi^{\prime}} \\
\frac{\partial E}{\partial \xi^{\prime}} & =\frac{16 \gamma \cos \theta}{\cos ^{2} \theta-4 \gamma k d} \zeta \zeta^{*}+\frac{4 \cos ^{3} \theta}{\cos ^{2} \theta-4 \gamma k d} \frac{1}{\pi} P \int_{-\infty}^{\infty} \frac{d \xi^{\prime \prime}}{\xi^{\prime \prime}-\xi^{\prime}} \frac{\partial}{\partial \xi^{\prime \prime}}\left(\zeta \zeta^{*}\right) .
\end{aligned}
$$


Substituting the expression for $\partial E / \partial \xi^{\prime}$ given by (35) in (34) we get the following single nonlinear evolution equation for $\zeta$ in the presence of a thin thermocline, and in this equation we drop the prime on $\xi$.

$$
i \frac{\partial \zeta}{\partial \tau}+\beta_{1} \frac{\partial^{2} \zeta}{\partial \xi^{2}}+i \beta_{2} \frac{\partial^{3} \zeta}{\partial \xi^{3}}=\Lambda_{1} \zeta^{2} \zeta^{*}+i \Lambda_{2} \zeta \zeta^{*} \frac{\partial \zeta}{\partial \xi}+i \Lambda_{3} \zeta^{2} \frac{\partial \zeta^{*}}{\partial \xi}+\Lambda_{4} \zeta H \frac{\partial}{\partial \xi}\left(\zeta \zeta^{*}\right)
$$

where

$$
\begin{gathered}
\beta_{1}=-\frac{1}{8}\left(\cos ^{2} \theta-2 \sin ^{2} \theta\right), \quad \beta_{2}=-\frac{1}{16}\left(\cos ^{3} \theta-6 \cos \theta \sin ^{2} \theta\right), \\
\Lambda_{1}=2+\frac{8 \gamma \cos ^{2} \theta}{\cos ^{2} \theta-4 \gamma k d}, \quad \Lambda_{2}=-6 \cos \theta, \\
\Lambda_{3}=-\cos \theta, \quad \Lambda_{4}=2+\frac{2 \cos ^{4} \theta}{\cos ^{2} \theta-4 \gamma k d}
\end{gathered}
$$

and the Hilbert transform operator $H$ is given by

$$
H \psi=\frac{1}{\pi} P \int_{-\infty}^{\infty} \frac{\psi\left(\xi^{\prime}\right)}{\xi^{\prime}-\xi} d \xi^{\prime}
$$

As the coefficients $\Lambda_{1}$ and $\Lambda_{2}$ contain the factor $\cos ^{2} \theta-4 \gamma k d$ in their denominators, the evolution equation (36) does not remain valid when $\cos ^{2} \theta=4 \gamma k d$, which is the resonance condition. This resonance condition is satisfied when the component of group velocity of the surface gravity wave along a line making an angle $\theta$ with the direction of propagation of the wave becomes equal to the phase velocity of the long wavelength internal wave. Moreover since the evolution equation (28) has been derived on the assumption that $k d$ is finite, results for $k d \rightarrow \infty$ cannot be obtained from the evolution equation (36).

\section{Stability of a uniform wave train}

For a uniform wave train we take

$$
\zeta=a_{0} \exp (-i \Delta \omega \tau) \equiv \zeta^{(0)}
$$

as the solution of (36), where $a_{0}$ is a real constant and $\Delta \omega$ is the nonlinear frequency shift of the wave given by

$$
\Delta \omega=\Lambda_{1} a_{0}^{2} .
$$


To study instability of the uniform wave train (39) we introduce the following perturbation in the uniform solution:

$$
\zeta=\zeta^{(0)}[1+\hat{\zeta}(\xi, \tau)]
$$

We substitute (41) in the evolution equation (36), linearize with respect to $\hat{\zeta}, \hat{\zeta}^{*}$ and then separate the equation into real and imaginary parts after setting $\hat{\zeta}=\hat{\zeta}_{r}+i \hat{\zeta}_{i}$, where $\hat{\zeta}_{r}, \hat{\zeta}_{i}$ are real. Finally taking Fourier transforms of these two equations with respect to $\xi$ we get the following two equations.

$$
\begin{gathered}
{\left[-\frac{\partial\left(\hat{\zeta}_{i}\right)}{\partial \tau}+i\left\{\beta_{2} l^{3}+\Lambda_{2} a_{0}^{2} l-l \Lambda_{3} a_{0}^{3}\right\}\left(\hat{\zeta}_{i}\right)\right]+\left[-\beta_{1} l^{2}-2 \Lambda_{1} a_{0}^{2}+2 \Lambda_{4} a_{0}^{2}|l|\right]\left(\hat{\zeta}_{r}\right)=0} \\
-\beta_{1} l^{2}\left(\hat{\zeta}_{i}\right)+\left[\frac{\partial\left(\hat{\zeta}_{r}\right)}{\partial \tau}-i\left\{l^{3} \beta_{2}+l \Lambda_{2} a_{0}^{2}-l \Lambda_{3} a_{0}^{3}\right\}\left(\hat{\zeta}_{r}\right)\right]=0
\end{gathered}
$$

where $\left(\hat{\zeta}_{r}\right)$ and $\left(\hat{\zeta}_{i}\right)$ denote Fourier transforms of $\hat{\zeta}_{r}$ and $\hat{\zeta}_{i}$ respectively with respect to $\xi$ defined by

$$
\left(\hat{\zeta}_{r, i}\right)=\frac{1}{\sqrt{2 \pi}} \int_{-\infty}^{\infty} \hat{\zeta}_{r, i} e^{-i l \xi} d \xi
$$

Now assuming $\tau$ dependence of $\left(\hat{\zeta}_{r}\right)$ and $\left(\hat{\zeta}_{i}\right)$ to be of the form $\exp (-i \Omega \tau)$ we get the following nonlinear dispersion relation from (42), (43).

$$
\Omega=-\beta_{2} l^{3}-\Lambda_{2} a_{0}^{2} l \pm\left[\beta_{1} l^{2}\left(\beta_{1} l^{2}+2 \Lambda_{1} a_{0}^{2}-2 \Lambda_{4} a_{0}^{2}|l|\right]^{1 / 2},\right.
$$

where we have neglected order $\epsilon^{5}$ terms, since the evolution equation (36), from which we have derived this nonlinear dispersion relation is correct to order $\epsilon^{4}$ terms.

The instability condition, that can be obtained from (60), is

$$
\beta^{2} l^{4}-2 \beta l^{2} a_{0}^{2}\left(\Lambda_{1}-\Lambda_{4}|l|\right)<0,
$$

where we have set $\beta_{1}=-\beta$. If this condition is satisfied the growth rate of the instability is given by

$$
\gamma_{1}=\left[2 \beta l^{2} a_{0}^{2}\left(\Lambda_{1}-\Lambda_{4}|l|\right)-\beta^{2} l^{4}\right]^{1 / 2} .
$$

This growth rate of instability attains a maximum when the wavenumber of the perturbation $l_{M}$ is given by

$$
l_{M}=\sqrt{\frac{\Lambda_{1}}{\beta}} a_{0}-\frac{3 \Lambda_{4}}{4 \beta} a_{0}^{2}
$$


and the corresponding maximum growth rate of instability $\gamma_{M}$ is given by

$$
\gamma_{M}=\Lambda_{1} a_{0}^{2}\left(l-\frac{\Lambda_{4}}{\sqrt{\beta \Lambda_{1}}} a_{0}\right) .
$$

According to (46) the wave number at marginal stability is given by

$$
\beta^{2} l^{2}-2 \beta a_{0}^{2}\left(\Lambda_{1}-\Lambda_{4}|l|\right)=0
$$

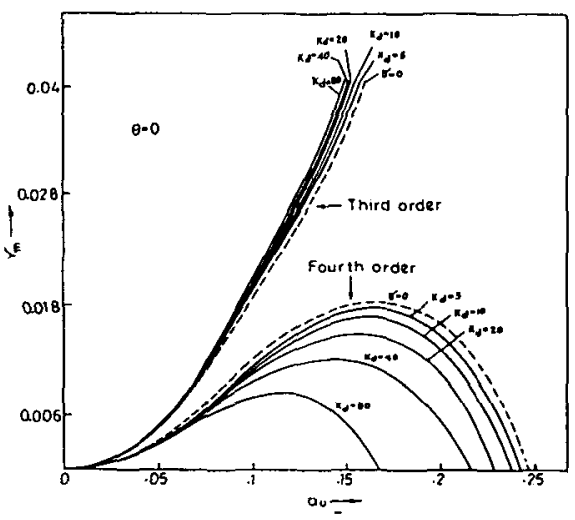

(a)

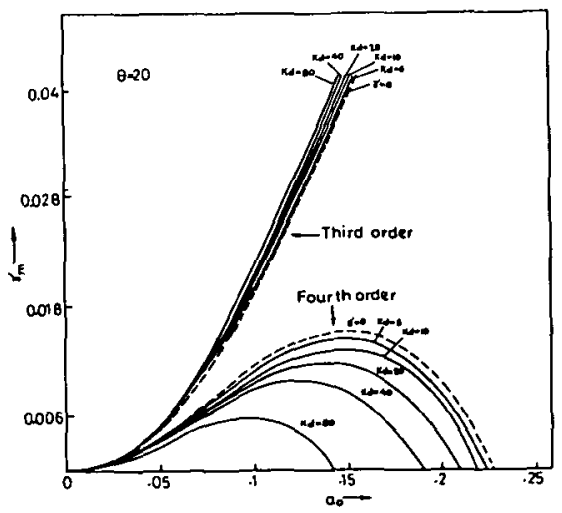

(c)

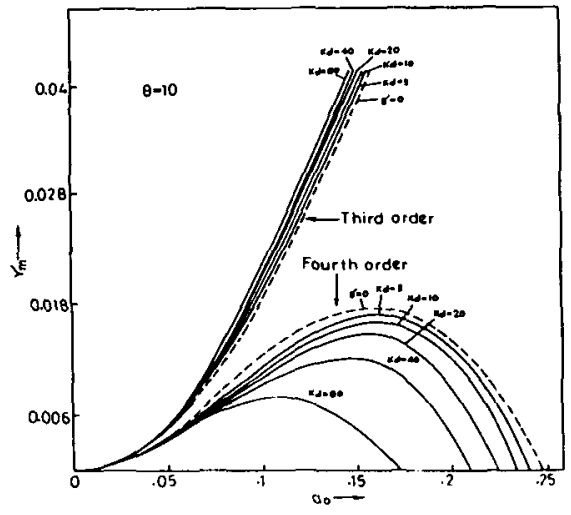

(b)

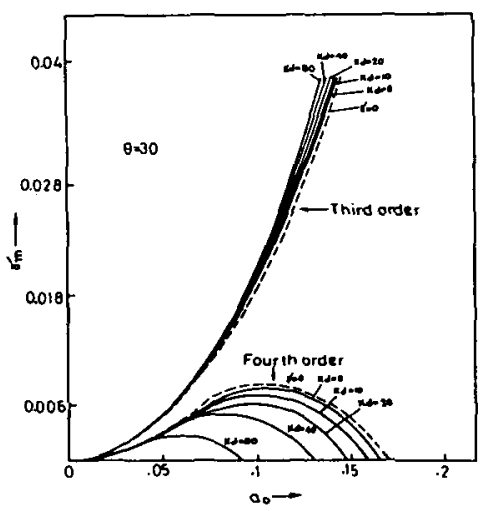

(d)

FIGURE 1. Maximum growth rate $\gamma_{M}$ against wave steepness $a_{0}, \ldots$ represents absence of thermocline.

For drawing graphs of Figures (1a)-(1d) and (2a)-(2d) we have taken $\gamma=10^{-3}$, which is the case for a seasonal thermocline. Graphs are plotted showing the variation of maximum growth rate of instability $\gamma_{M}$ against wave steepness $a_{0}$ for 5 different values of $k d$ and for a fixed value of $\theta$. Figures (1a)-(1d) are drawn respectively 


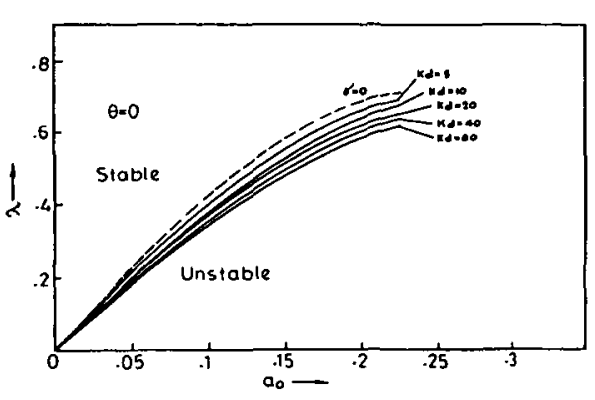

(a)

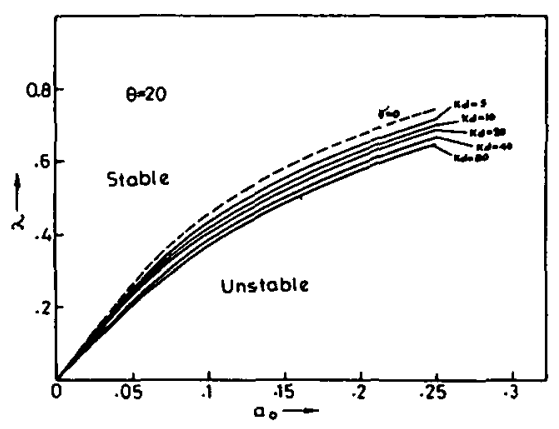

(c)

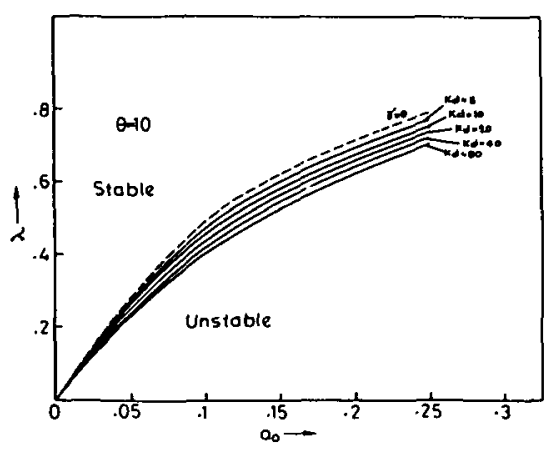

(b)

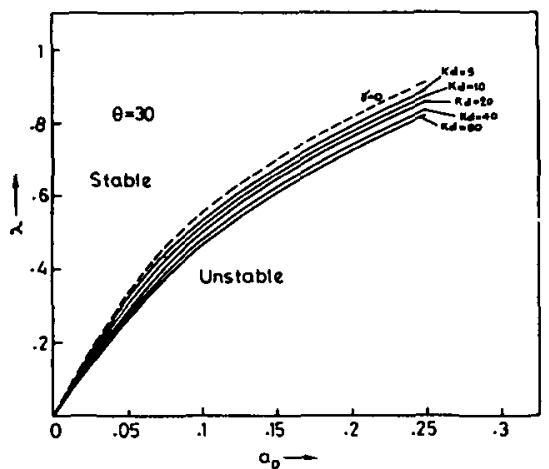

(d)

FlGURE 2. Wave number of perturbation $\lambda$ at marginal stability against wave steepness $a_{0}$, ---represents absence of thermocline.

for $\theta=0^{\circ}, 10^{\circ}, 20^{\circ}, 30^{\circ}$. In each of the figures graphs of maximum growth rate of instability obtained from the third-order evolution equation are shown; moreover the graphs for maximum growth rate of instability in the absence of a thermocline $(\gamma=0)$ obtained from the fourth-order evolution equation are also shown. From these graphs it is found that a thin thermocline has a stabilizing influence on the instability of a uniform surface - gravity wave train and the maximum growth rate of instability decreases with the increase of thermocline depth. From this it cannot of course be concluded that the maximum growth rate of instability tends to zero, that is, there is stability as $k d \rightarrow \infty$. Since as mentioned in the last paragraph of Section 3 the results for $k d \rightarrow \infty$ cannot be obtained from evolution equation (36).

The wavenumber of perturbation $\lambda$ at marginal stability has been plotted against wave steepness $a_{0}$ showing stable-unstable regions in $\lambda a_{0}$ - space for five different values of $k d$ in four figures (2a)-(2d), which are drawn respectively for $\theta=0^{\circ}, 10^{\circ}, 20^{\circ}, 30^{\circ}$.

In figures (3a)-(3d) graphs have been plotted showing the variations of maximum 


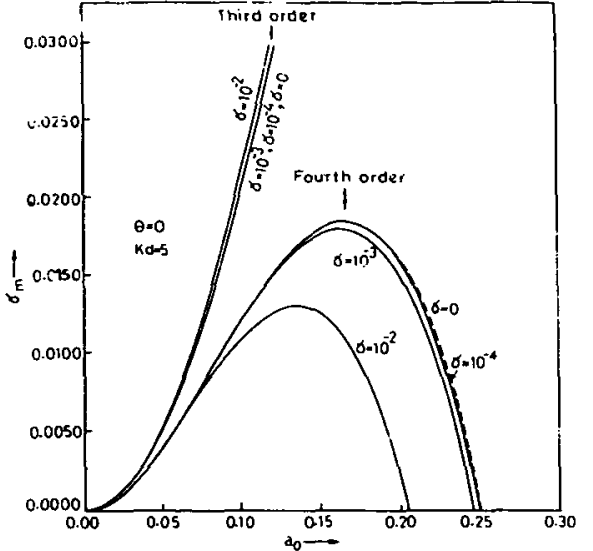

(a)

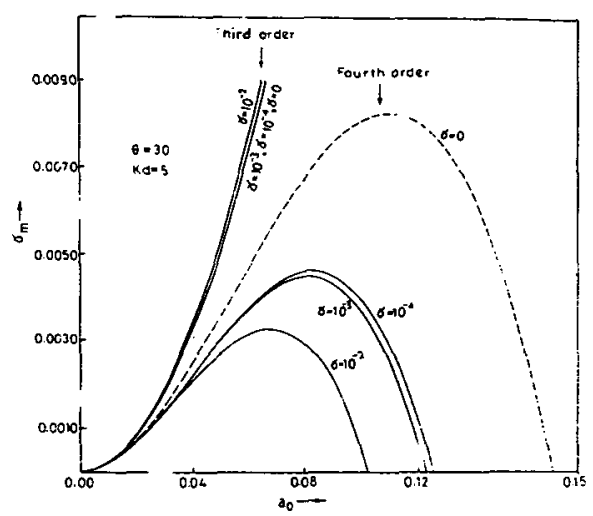

(c)

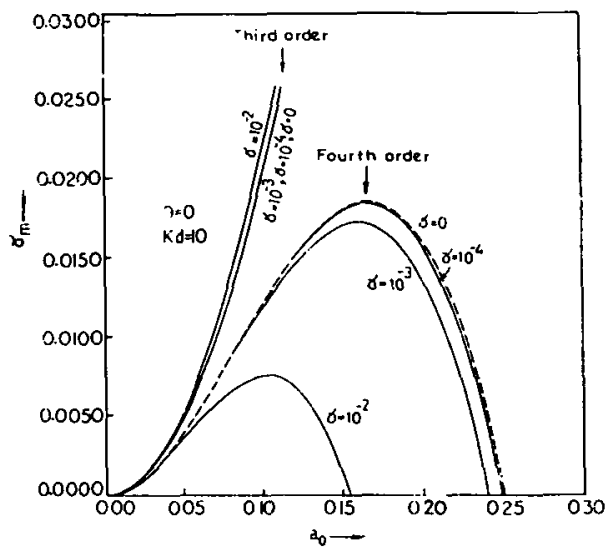

(b)

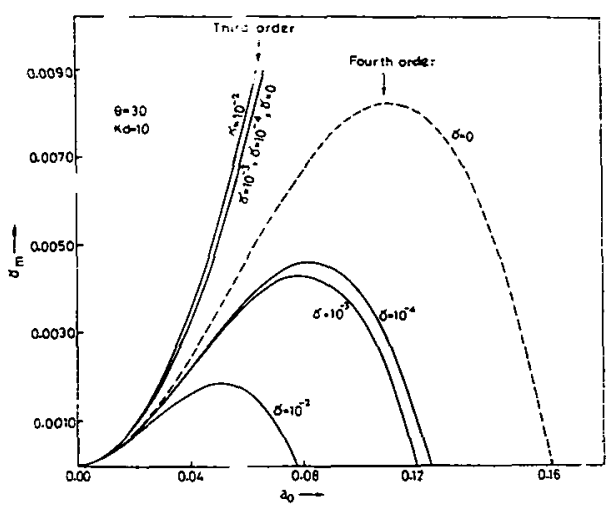

(d)

FIGURE 3. Maximum growth rate $\gamma_{M}$ against wave steepness $a_{0}$, ---- represents absence of thermocline.

growth rate of instability $\gamma_{M}$ against wave-steepness $a_{0}$ for four different values of $\gamma$ and for some fixed values of $k d$ and $\theta$.

\section{Conclusion}

In order to study the effect of a thin thermocline on the Benjamin-Feir instability of a surface gravity wave, we have derived two coupled fourth-order nonlinear evolution equations. The reason for starting from fourth-order nonlinear evolution equations is that for infinite-depth fluids as pointed out by Dysthe [8] the fourth-order nonlinear evolution equations give results consistent with the exact results of Longuet-Higgins $[15,16]$ for wave steepness up to 0.25 . Assuming that space variation of amplitude 
takes place along an arbitrary fixed line, the two coupled equations are reduced to a single equation. From this equation instability condition and maximum growth rate of instability are obtained. It is found that a thin thermocline has a stabilizing influence and the maximum growth rate of instability decreases with the increase of thermocline depth. These results remain valid only for finite thermocline depth, since the evolution equations have been derived under this condition. Further the single evolution equation loses its validity when the resonance condition is satisfied. This resonance condition is satisfied when the component of group velocity of the surface gravity wave along the line in which the perturbation is given becomes equal to the phase velocity of the long internal wave. Both the maximum growth rate of the instability and wavenumber at marginal stability have been shown graphically for some different values of dimensionless thermocline depth.

\section{Acknowledgements}

The authors acknowledge the helpful comments of the referees.

\section{Appendix}

$$
\begin{aligned}
\Delta_{1} A_{1}+i\left(\omega-i \epsilon C_{g} \frac{\partial}{\partial \xi}+i \epsilon^{2} \frac{\partial}{\partial \tau}\right) \zeta_{1} & =a_{1}, \\
-i\left(\omega-i \epsilon C_{g} \frac{\partial}{\partial \xi}+i \epsilon^{2} \frac{\partial}{\partial \tau}\right) A_{1}+g \zeta_{1} & =b_{1}, \\
\Delta_{2} A_{2}+i\left(2 \omega-i \epsilon C_{g} \frac{\partial}{\partial \xi}+i \epsilon^{2} \frac{\partial}{\partial \tau}\right) \zeta_{2} & =a_{2}, \\
-i\left(2 \omega-i \epsilon C_{g} \frac{\partial}{\partial \xi}+i \epsilon^{2} \frac{\partial}{\partial \tau}\right) A_{2}+g \zeta_{2} & =b_{2},
\end{aligned}
$$

where $a_{n}, b_{n},(n=1,2)$ are contributions from nonlinear terms.

$$
\begin{gathered}
\epsilon \bar{k} \bar{F}-i \epsilon C_{g} k_{\xi} \bar{\zeta}_{0}-\epsilon^{2} \frac{\partial \bar{\zeta}_{0}}{\partial \tau}=\bar{a}_{0}, \\
i \epsilon C_{g} k_{\xi} \bar{E}+g \bar{\zeta}_{0}+\epsilon^{2} \frac{\partial \bar{E}}{\partial \tau}=\bar{b}_{0},
\end{gathered}
$$

where $\bar{E}=\bar{A}_{0}+\bar{B}_{0}, \bar{F}=\bar{A}_{0}-\bar{B}_{0}$, and a bar over $a_{0}, b_{0}$ indicates their Fourier transform.

$$
\bar{A}_{0} e^{-\epsilon \bar{k} d}-\bar{B}_{0} e^{\epsilon \bar{k} d}=\bar{C}_{0} e^{-\epsilon \bar{k} d}
$$




$$
\begin{aligned}
& -\epsilon^{2} k_{\xi}^{2} C_{g}^{2} \bar{k}^{2}\left(\bar{A}_{0} e^{-\epsilon \bar{k} d}+\bar{B}_{0} e^{\epsilon \bar{k} d}-\bar{C}_{0} e^{-\epsilon \bar{k} d}\right) \\
& =\frac{\epsilon g \delta \rho \bar{k}^{3}}{\rho}\left(\bar{A}_{0} e^{-\epsilon \bar{k} d}-\bar{B}_{0} e^{\epsilon \bar{k} d}\right)-2 i \epsilon^{3} C_{g} k_{\xi} \bar{k}^{2}\left(\frac{\partial \bar{A}_{0}}{\partial \tau} e^{-\epsilon \bar{k} d}+\frac{\partial \bar{B}_{0}}{\partial \tau} e^{\epsilon \bar{k} d}-\frac{\partial \bar{C}_{0}}{\partial \tau} e^{-\epsilon \bar{k} d}\right), \\
& -\epsilon^{2} C_{g}^{2} \bar{k}^{2} k_{\bar{\xi}}^{2}(\bar{E}-\bar{F}) e^{\epsilon \bar{k} d} \\
& =\frac{\epsilon g \delta \rho \bar{k}^{3}}{\rho}(\bar{F} \cosh \epsilon \bar{k} d-\bar{E} \sinh \epsilon \bar{k} d)-2 i \epsilon^{3} C_{g} k_{\xi} \bar{k}^{2}\left(\frac{\partial \bar{E}}{\partial \tau}-\frac{\partial \bar{F}}{\partial \tau}\right) e^{\epsilon \bar{k} d} .
\end{aligned}
$$

Solutions for $\zeta_{i j}, A_{i j}$ and $F_{j}$.

$$
\begin{aligned}
A_{11}= & -\frac{i \omega}{k} \zeta_{11}, \quad A_{12}=-\frac{i \omega}{k} \zeta_{12}+\frac{1}{k^{2}}\left(\omega-k C_{g}\right) \frac{\partial \zeta_{11}}{\partial \xi}, \\
A_{22}= & 0, \quad A_{23}=\frac{1}{k}\left(2 k C_{g}-\omega\right) \zeta_{11} \frac{\partial \zeta_{11}}{\partial \xi}, \\
\zeta_{22}= & k \zeta_{11}^{2}, \quad \zeta_{23}=2 k \zeta_{11} \zeta_{12}+\frac{2 i}{\omega}\left(k C_{g}+\omega\right) \zeta_{11} \frac{\partial \zeta_{11}}{\partial \xi}, \\
\zeta_{02}= & \frac{C_{g}}{g} \frac{\partial E_{1}}{\partial \xi}, \quad \zeta_{03}=\frac{C_{g}}{g} \frac{\partial E_{2}}{\partial \xi}-\frac{1}{g} \frac{\partial E_{1}}{\partial \tau}, \\
F_{2}= & -\frac{C_{g}^{2}}{g} F^{-1}\left[\frac{1}{\bar{k}} F\left[\frac{\partial^{2} E_{1}}{\partial \xi^{2}}\right]\right]+2 \omega F^{-1}\left[\frac{1}{\bar{k}} F\left[\frac{\partial}{\partial \xi}\left(\zeta_{11} \zeta_{11}^{*}\right)\right]\right] \\
F_{3}= & \left.\left.-\frac{C_{g}^{2}}{g} F^{-1}\left[\frac{1}{\bar{k}} F\left[\frac{\partial^{2} E_{2}}{\partial \xi^{2}}\right]\right]+\frac{2 C_{g}}{g} F^{-1}\left[\frac{1}{\bar{k}} F\right] \frac{\partial^{2} E_{1}}{\partial \xi \partial \tau}\right]\right] \\
& +i C_{g} F^{-1}\left[\frac{1}{\bar{k}} F\left[\frac{\partial}{\partial \xi}\left(\zeta_{11} \frac{\partial \zeta_{11}^{*}}{\partial \xi}-\zeta_{11}^{*} \frac{\partial \zeta_{11}}{\partial \xi}\right)\right]\right] \\
& +\frac{i \omega}{k} F^{-1}\left[\frac{1}{\bar{k}} F\left[\frac{\partial}{\partial \eta}\left(\zeta_{11} \frac{\partial \zeta_{11}^{*}}{\partial \eta}-\zeta_{11}^{*} \frac{\partial \zeta_{11}}{\partial \eta}\right)\right]\right] \\
& +2 \omega F^{-1}\left[\frac{1}{\bar{k}} F\left[\frac{\partial}{\partial \xi}\left(\zeta_{12} \zeta_{11}^{*}+\zeta_{11} \zeta_{12}^{*}\right)\right]\right]
\end{aligned}
$$

Here $F^{-1}[$ ] implies Fourier inversion of the quantity inside bracket.

Derivation of the evolution equations from (A2) and (A1). (A2) can be put in the following convenient form after eliminating $A_{1}$ by the use of (A1).

$$
\left[\left(\omega-i \epsilon C_{g} \frac{\partial}{\partial \xi}+i \epsilon^{2} \frac{\partial}{\partial \tau}\right)^{2}-g \Delta_{1}\right] \zeta_{1}=-\Delta_{1} b_{1}-i\left(\omega-i \epsilon C_{g} \frac{\partial}{\partial \xi}+i \epsilon^{2} \frac{\partial}{\partial \tau}\right) a_{1}
$$

This equation in the lowest order, which is order $\epsilon^{3}$, becomes the following after 
substituting the solution for various perturbed quantities.

$$
i \frac{\partial \zeta_{11}}{\partial \tau}-\frac{\omega}{8 k^{2}} \frac{\partial^{2} \zeta_{11}}{\partial \xi^{2}}+\frac{\omega}{4 k^{2}} \frac{\partial^{2} \zeta_{11}}{\partial \eta^{2}}=2 \omega k^{2} \zeta_{11}^{2} \zeta_{11}^{*}+k \zeta_{11} \frac{\partial E_{1}}{\partial \xi} .
$$

At the next order, $O\left(\epsilon^{4}\right)$, the same equation (A11) gives

$$
\begin{aligned}
i \frac{\partial \zeta_{12}}{\partial \tau}- & \frac{\omega}{8 k^{2}} \frac{\partial^{2} \zeta_{12}}{\partial \xi^{2}}+\frac{\omega}{4 k^{2}} \frac{\partial^{2} \zeta_{12}}{\partial \eta^{2}}-\frac{i \omega}{16 k^{3}} \frac{\partial^{3} \zeta_{11}}{\partial \xi^{3}}+\frac{3 i \omega}{8 k^{3}} \frac{\partial^{3} \zeta_{11}}{\partial \xi \partial \eta^{2}} \\
= & 2 \omega k^{2}\left(\zeta_{11}^{2} \zeta_{12}^{*}+2 \zeta_{11} \zeta_{11}^{*} \zeta_{12}\right)-5 i \omega k \zeta_{11} \zeta_{1}^{*} \frac{\partial \zeta_{11}}{\partial \xi} \\
& -i \omega k\left(\zeta_{11} \zeta_{11}^{*} \frac{\partial \zeta_{11}}{\partial \xi}+\zeta_{11}^{2} \frac{\partial \zeta_{11}^{*}}{\partial \xi}\right)+k\left(\zeta_{11} \frac{\partial E_{2}}{\partial \xi}+\zeta_{12} \frac{\partial E_{1}}{\partial \xi}\right)
\end{aligned}
$$

In this equation we have replaced $\partial \zeta_{11} / \partial \tau$, by the expression that can be obtained from (A12).

Adding (A12) and (A13) and setting $\zeta=\zeta_{11}+\epsilon \zeta_{12}, E=E_{1}+\epsilon E_{2}$ we arrive at the fourth-order nonlinear evolution equation (27) for $\zeta$. This equation has been made dimensionless by introducing dimensionless quantities in bars given by (30) and then finally dropping bars.

Since the evolution equation (27) involves $E$, we need an equation for $E$. This is provided by the equation (A9), which in the lowest order, $O\left(\epsilon^{3}\right)$ gives the equation

$$
\left(k_{\zeta}^{2} C_{g}^{2}-\frac{g \delta \rho}{\rho} \bar{k}^{2} d\right) \bar{E}_{1}=-\frac{2 g \omega \delta \rho}{\rho} F\left[\frac{\partial}{\partial \xi} \zeta_{11} \zeta_{11}^{*}\right],
$$

where $F[]$ implies the Fourier transform of the quantity inside the square bracket. At the next order, that is, at order $\epsilon^{4}$ the same equation gives

$$
\left(k_{\zeta}^{2} C_{g}^{2}-\frac{g \delta \rho}{\rho} \bar{k}^{2} d\right) \bar{E}_{2}=\frac{2 \omega C_{g}^{2} k_{\xi}^{2}}{\bar{k}} F\left[\frac{\partial}{\partial \xi} \zeta_{11} \zeta_{11}^{*}\right],
$$

where we have neglected terms of order $\epsilon^{4} \delta \rho / \rho$.

Now adding (A14) and (A15) then taking the Fourier inversion, we get (28) for $E$, which has been written in dimensionless form after introducing the dimensionless quantities (30) and then dropping their bars.

\section{References}

[1] F. K. Ball, "Energy transfer between external and internal waves", J. Fluid. Mech. 19 (1964) 465-478. 
[2] U. Brinch-Nielsen and I. G. Jonsson, "Fourth order evolution equations and stability analysis for Stokes waves on arbitrary water depth", Wave Motion 8 (1986) 455-472.

[3] K. P. Das, "On evolution equations for a three dimensional surface gravity wave packet in a two layer fluid", Wave Motion 8 (1986) 191-204.

[4] A. Davey and K. Stewartson, "On three-dimensional packets of surface waves", Proc. $R$. Soc. Lond. A 338 (1974) 101-110.

[5] A. K. Dhar and K. P. Das, "A fourth-order evolution equation for deep water surface gravity waves in the presence of wind blowing over water", Phys. Fluids A 2 (1990) 778-783.

[6] A. K. Dhar and K. P. Das, "Fourth order nonlinear evolution equation for two Stokes wave trains in deep water", Phys. Fluids A 3 (1991) 3021-3026.

[7] A. K. Dhar and K. P. Das, "Stability analysis from fourth order evolution equation for small but finite amplitude interfacial waves in the presence of a basic current shear", J. Austral. Math. Soc. $B 35$ (1994) 348-365.

[8] K. B. Dysthe, "Note on a modification to the nonlinear Schrödinger equation for application to deep water waves", Proc. R. Soc. Lond. A 369 (1979) 105-114.

[9] K. B. Dysthe and K. P. Das, "Coupling between a surface wave spectrum and an internal wave: modulational interaction", J. Fluid Mech. 104 (1981) 483-503.

[10] M. Funakoshi and M. Oikawa, "The resonant interaction between a long internal gravity wave and a surface gravity wave packet", J. Phys. Soc. Japan 52 (1983) 1982.

[11] T. Hara and C. C. Mei, "Frequency downshift in narrowbanded surface waves under the influence of wind", J. Fluid Mech. 230 (1991) 429-477.

[12] K. Hasselman, "Nonlinear interactions treated by method of theoretical physics (with application to generation of waves by wind)", Proc. R. Soc. Lond. A 229 (1967) 77.

[13] S. J. Hogan, "Fourth order evolution equation for deep water gravity-capillary waves", Proc. $R$. Soc. Lond. A 402 (1985) 359-372.

[14] P. A. E. M. Janssen, "On a fourth order envelope equation for deep water waves", J. Fluid Mech 126 (1983) 1-11.

[15] M. S. Longuet-Higgins, "The instabilities of gravity waves of finite amplitude in deep water. I Super harmonics", Proc. R. Soc. Lond. A 360 (1978) 471-488.

[16] M. S. Longuet-Higgins, "The instabilities of gravity waves of finite amplitude in deep water. II Subharmonics", Proc. R. Soc. Lond. A 360 (1978) 489-505.

[17] Y. C. Ma, "A study of resonant interaction between internal and surface waves based on a two layer fluid model", Wave Motion 5 (1983) 145.

[18] D. J. Olbers and K. Herterich, "The spectral energy transfer from surface waves to internal waves", J. Fluid Mech. 92 (1979) 349-379.

[19] O. M. Phillips, The Dynamics of the Upper Ocean (Cambridge University Press, 1977).

[20] M. H. Risk and D. R. S. Ko, "Interaction between small scale surface waves and large scale internal waves", Phys. Fluids 21 (1978) 1900-1907.

[21] M. Stiassnie, "Note on modified nonlinear Schrödinger equation for deep water waves", Wave Motion 6 (1984) 431-433.

[22] S. A. Thorpe, "On wave interaction in a stratified fluid", J. Fluid Mech. 24 (1966) 737.

[23] K. M. Watson, B. J. West and B. I. Cohen, "Coupling of surface and internal gravity waves: a mode coupling model", J. Fluid Mech. 77 (1976) 185. 